\title{
Consumidores de fármacos: \\ o que pensam e o que sabem?
}

\section{Medicine consumers: what do they believe, what do they know?}

\author{
Luiz Miguel Santiago ${ }^{1}$ \\ Salvador Massano Cardoso ${ }^{2}$
}

\section{Resumo}

$\mathrm{Na}$ sociedade atual, é cada vez mais marcante o papel da terapêutica farmacológica na atividade curativa desempenhada pelos médicos. A aquisição de medicamentos não sujeitos a receita médica é também hoje uma realidade em farmácia e em parafarmácia. É fonte de preocupação a segurança pela utilização de medicamentos, bem como o desenvolvimento de políticas equitativas de acesso a cuidados de saúde e a medicamentos. A utilização de medicamentos será tanto melhor realizada quanto melhor for o conhecimento dos utilizadores acerca do processo fármaco-terapêutico, resultando em ganhos de saúde e em melhoria de eficiência. Para tal, defende-se o estudo do conhecimento que o público detém sobre medicamentos, em particular no ambiente de Medicina Geral e Familiar, no qual o peso desta realidade deve ser medido.

\begin{abstract}
Pharmacological therapy is one of the most studied matters in public health and one of the most important issues in General Practice / Family Medicine. OTC medicines are a growing reality. Medicine safety as well as equity in the distribution of health care is still a matter of concern for our democratic societies. The more the user knows about medicines the better he will use them, resulting in economic and health gains. In the light of the above, it is important to study the knowledge of the public at large about medicines, in particular in the Primary Care setting, namely in Family Medicine, where such issues are of utmost importance.
\end{abstract}

Palavras-chave: Medicina Interna, Preparações Farmacêuticas; Farmácia; Economia Farmacêutica; Quimioterapia.
Key Words: Internal Medicine; Pharmacentical Preparations; Pharmacy; Economics; harmaceutical; Drug Therapy.

${ }^{1}$ Médico, Chefe de Serviço de Clínica Geral, Centro de Saúde de Eiras, ARS do Centro, Coimbra, Portugal.

${ }^{2}$ Professor catedrático e diretor do Instituto de Higiene e Medicina Social, Faculdade de Medicina, Universidade de Coimbra, Coimbra, Portugal. 


\section{Introdução}

Segundo o DL 176/2006 de 30 de Agosto', define-se medicamento como "toda a substância, ou associações de substâncias, apresentada como possuindo propriedades curativas ou preventivas de doenças em seres humanos ou dos seus sintomas ou que possa ser utilizada ou administrada no ser humano, a fim de estabelecer um diagnóstico médico ou, exercendo uma ação farmacológica, imunológica ou metabólica, restaurar, corrigir ou modificar funções fisiológicas" e medicamento à base de plantas como "qualquer medicamento que tenha exclusivamente como substâncias activas uma ou mais substâncias derivadas de plantas, uma ou mais preparações à base de plantas ou uma ou mais substâncias derivadas de plantas em associação a uma ou mais preparações à base de plantas".

A ingestão de medicamentos pode ter vários indutores $^{2}$, não parecendo ser desprezível a freqüência da automedicação, definida como a decisão de tomar medicamentos numa lógica não ditada pela indicação expressa de médico ou pela sua simples aquisição sem receita médica ${ }^{3,4}$. Num contexto de terapêutica analgésica a automedicação pode ser muito freqüente com todo o cortejo de problemas terapêuticos e de resultados em saúde que podem advir de medicamentos não referidos ao médico ${ }^{3,5}$. As questões da medicação segura são também valorizadas por quem adere à automedicação, sendo estas julgadas seguras pois que se encontram disponíveis em parafarmácia para aquisição direta ${ }^{6}$.

Existe hoje entre a classe médica reflexão aprofundada sobre a prescrição, sendo discutidos temas como a obrigatoriedade de prescrever para atingir orientações de relatórios contendo linhas de orientação $\mathrm{O}^{7,8,9,10,11}$, bem como sobre o tipo de medicamentos prescritos, questionando-se a sua eficiência ${ }^{12}$, assim como sobre se não haverá excesso de medicalização ${ }^{13}$.

Em Portugal, o Instituto Nacional da Farmácia e do Medicamento, a autoridade regulamentadora de medicamentos, decidiu difundir informação acerca dos perigos que a medicação de venda livre pode ter, mas a eficiência de tal medida estará ainda por se verificar ${ }^{14}$. São claras as regras, quer quanto aos locais de venda, ao tipo de medicamentos vendáveis e à estrutura de pessoal que trabalha em instalações de medicamentos não sujeitos a receita médica obrigatória ${ }^{15}$. Ainda segundo o Instituto Nacional da Farmácia e do Medicamento, a evolução recente do mercado de medicamentos não sujeitos a receita médica obrigatória revela maior volume de vendas para o grupo farmacoterapêutico "Analgésicos e Antipiréticos"16 pelo fato de Paracetamol e Ibuprofeno terem passado ao estatuto de medicamento não sujeito a receita médica obrigatória por apresentarem uma boa relação risco/benefício que obriga, no entanto, à contínua monitorização de segurança $a^{17}$. Tanto para o Paracetamol como para o Ibuprofeno são bem conhecidas as vias de metabolização e os eventuais concorrentes para aumento ou redução de tal metabolização com correspondente resultado terapêutico, quer sejam outros medicamentos ou nutrientes ${ }^{18,19}$.

Segundo a definição de Saúde da $\mathrm{OMS}^{20}$, o estado de saúde será difícil de existir na sua plenitude. No entanto, a sociedade atual deve preocupar-se não só com a saúde para todos, segundo Alma Ata ${ }^{21}$, como também por responsabilizar os indivíduos pelo seu estado de saúde, levando-os a adotar os melhores estilos de vida para a obtenção do melhor estado, por vezes à custa de alguns sacrifícios individuais, fruto da obtenção de aconselhamento técnico que deverá ser obtido em sistemas organizados e funcionantes, quer oficiais quer particulares, que os estados devem prover que funcionem como as Declarações de Sundsvall e Jacarta ${ }^{22,23,24}$ propõem.

No entanto, a informação contemporânea e a globalização impõem modelos de vida e de consumo de bens que se coadunam mal e são até mesmo conflitantes com o bom estado de saúde ${ }^{22,23,24}$. A informação pública, via órgãos de comunicação escrita, visual ou falada, ao definir padrões, impôr conceitos e orientar para táticas conducentes à estratégia do bem-estar, pode determinar excessiva medicalização da sociedade ${ }^{25}$.

De fato, a simplificação da realidade, criando modelos estereotipados de repetição, tipificação e esquematização, pode desencadear situações em que o próprio julga 
a decisão e assume o padrão de consumo ${ }^{25}$. O conhecimento através da oralidade e transmissão longitudinal de conhecimentos foi, há tempos - e ainda o é hoje -, forma importante de manutenção de técnicas de "cura e prevenção" um pouco em contraponto à massificação transversal que hoje se verifica ${ }^{25}$. Estando quem se sente doente inserido num sistema, a comunicação, seja ela de que tipo for, passa a determinar os comportamentos daqueles que interagem, sendo de particular interesse o modo como flui e a forma como é apresentada a informação sobre o que é, o que se faz e o que está ao alcance individual para moldar uma forma de bem-estar que o corpo individual deseja, pois se parte do princípio de que a sociedade e os bens são talhados para a felicidade individual, podendo e devendo ser acedidos sempre que a sua necessidade é sentida ${ }^{25}$.

Neste contexto, a Comissão Européia assumiu recentemente posição ao editar o relatório Future challenges paper: 2009-2014 ${ }^{26}$, em que, pensando em quatro orientadores gerais de orientação para o qüinqüênio - a governância, a confiança, a sociedade em mudança e a globalização -, considera haver 13 fatores críticos a atender. Dentre eles, consideram-se de especial importância para o tema em apreço:

- A apresentação de resultados "reais".

- A adesão a um sistema de abertura e transparência.

- A compreensão da visão de risco do consumidor.

- O eficiente uso e partilha de conhecimento.

- A monitorização da crescente complexidade dos comportamentos dos consumidores.

- A importância dos prestadores de serviço serem uma fonte de informação credível.

- A compreensão de novas e adicionais influências nas escolhas dos consumidores.

A União Européia está, assim, preocupada com a qualidade dos cuidados de saúde a pacientes, tendo aprovado a Declaração de Luxemburgo, em 2005, com recomendações às Instituições da União Européia, às Autoridades Nacionais e aos prestadores de cuidados de saúde, tratando de lidar com os erros da medicalização vendo-os como oportunidades de aprendizagem e experimentando melhores soluções de relacionamento médicos/doentes ${ }^{27}$.

Os conceitos de doença, estar doente e sentir-se mal como estarão a orientar a necessidade de consumir medicamentos? E como se compreenderá, assim, o medicamento: um bem de consumo normal, um meio de cura e como tal passível de causar dano também? ${ }^{28}$

O empowerment dado aos cidadãos estará a ser bem realizado $^{29}$ ? Saberão os utilizadores do medicamento o poder que estes lhes dão ${ }^{30,31}$ ? Sentir-se-ão melhor por ter tal poder? Muita informação lhes é disponibilizada, muitas novidades científicas lhes são confiadas e, por vezes, de valia e resultados a curto prazo questionáveis ${ }^{32}$. Em particular a crianç $^{32}$ e o idoso ${ }^{33}$ são alvos de muitos cuidados, informação e terapêutica que devem ser questionados constantemente, até por serem pensados para o seu "bem-estar", mas incorporando noções em que o corpo pode sofrer pelo excesso de manipulação física ou química que, pretendendo um resultado, acaba por obter outro bem diferente ${ }^{17}$.

A nível de estruturas enquadradoras da atividade médica na Europa, é defendido para os médicos de Clínica Geral/Medicina Familiar, o papel de advocacia da saúde dos seus clientes que se consubstancia nos melhores enquadramentos diagnósticos, de terapêuticos e de referenciação. Tal implica forçosamente o melhor conhecimento das terapêuticas a serem realmente efetuadas pelos pacientes, incluindo a automedicação, seja ela artesanal ou empírica ${ }^{34}$.

A excessiva medicalização da sociedade pode ser vista do lado médico como a necessidade de a todo o custo encontrar doenças, mesmo que apenas haja sintomas para assim utilizar tecnologia que lhe pode trazer bem-estar social e pessoal, em particular medicamentosa ${ }^{35}$.

Do lado do paciente é interessante pensar que a população, em geral leiga quanto a questões de diagnóstico e terapêutica, mas com grande necessidade de ganhos próprios, familiares ou sociais, pela patologia vê a medicina como uma "arte" de descobrir doenças, bem caracterizado em textos jornalísticos, que mostram ou refletem sobre formas completamente diferentes de ver este processo, que não apenas o lado técnico daqueles ligados à Medicina e 
que, por força da sua difusão pública, são capazes de interferir e formatar opiniões e crenças acerca desta temática como os da autoria de António José Saraiva, em "O Sol”36, e de Miguel Sousa Tavares, em "Expresso", de 2 de Fevereiro de 2008, em página sete, do caderno principal.

Assim, podemo-nos inquirir sobre se os pacientes foram informados na consulta da necessidade de tomar medicamentos e sobre se estarão a querer tomar medicamentos para mitigarem a sua responsabilidade de não cumprimento das regras mais básicas de cidadania: respeito por si próprios mantendo adequados estilos de vida saudável ${ }^{21,22,23}$ ?

Estará quem procura medicamentos dependente destes ou já "habituado"?

Saberão os doentes que no processo farmacocinético há também interacções com alimentos e nutrientes ${ }^{23,24}$ ?

O processo terapêutico implica uma cadeia de muitos elos e que poderemos teorizar, esquematicamente, como os seguintes:

Boa colheita de dados sobre um paciente para a produção de um quadro sobre o qual trabalhar.

Base sólida de conhecimentos de farmacologia pelos médicos e na capacidade de transmissão de informação sobre a necessidade de induzir terapêutica, pela sua explicação e concordância e aquiescência pelo doente sobre as regras.

Correta cedência de medicamentos.

Administração do medicamento pelo doente que, além de o ingerir, o metaboliza e elimina em processos que dependem também de interações várias ${ }^{37,38}$.

Podemos também pensar que este processo decorre, em relação aos medicamentos sujeitos a receita médica obrigatória, numa necessidade de atividade sinérgica entre prescritor e farmacêutico - e outros profissionais em outros contextos nacionais - que, em ambiente de farmácia de oficina, cede ou dispensa o medicamento. Parece verificar-se entre estes dois profissionais alguns problemas que devem ser atendidos ${ }^{39}$. Questão, por vezes de difícil resolução, é em muitos casos o critério seguido aquando da substituição de medicamento na farmácia por razões de operação do sistema, podendo não estar claras as razões para tal ${ }^{39}$.

Continuamos a ver refletida insistentemente a questão da informação sem que tenha até agora sido questionado, do lado do paciente que tem de tomar medicamentos, o que sobre eles ele sabe, sabendo-se que o custo da terapêutica é importante para a adesão e manutenção em seguimento, particularmente para os mais idosos e em terapêuticas de longa duração ${ }^{40}$, como no particular contexto do Brasil. Ao mesmo tempo, continuamos a saber, para Portugal, de baixos valores de controle terapêutico como no caso da hipertensão arterial ${ }^{41}$.

Estudos metodologicamente corretos e apresentados na Conferência Nacional de Economia de Saúde, edições IX e X, elaborados para o total nacional, particularizando-o por regiões, permitem verificar um valor muito elevado, quer em volume, quer em valor de desperdício em medicamentos - definido como quantidade de embalagens não-consumidas -, situando-se em 4,44 euros por medicamento, leia-se caixa de medicamento, representando o cofinanciamento do Serviço Nacional de Saúde 60,4\% do total dos encargos desperdiçados ${ }^{42}$. Também, por classes terapêuticas, é verificável desperdício, quer para analgésicos e antiinflamatórios não-esteróides, quer para anti-histamínicos e antibióticos com valores muito significativos ${ }^{43}$. Ainda segundo o relatório de atividades de 2006 da Valor $\mathrm{Med}^{44}$, entidade creditada para a recolha e tratamento de resíduos de medicamentos, foram recolhidos, em 2006, 578 toneladas de medicamentos e, em 2005, 471 toneladas, o que significa um acréscimo de 22,7\%, apesar de, de 2005 para 2006, o número de farmácias aderentes ter subido de 2.709 para $2.744(+1,3 \%)$.

Em outros contextos, foi já descrito ser fundamental a informação/formação realizada a cada indivíduo pelo seu médico prescritor, que acaba por ser, de fato, a fonte de informação sobre medicamentos como percebido em estudo neo-zelandês ${ }^{45}$ e que foi vertida em forma de relatório pela preocupação existente com a não-adesão ou o nãocumprimento das terapêuticas pelos pacientes, resultando em fracos resultados pela terapêutica farmacológica, apesar das elevadas somas financeiras investidas por terceiros 
pagadores nos medicamentos ${ }^{46}$.

A comunicação, para a mais adequada terapêutica, deve ser bidirecional, pois é importante que o médico possa ser intérprete das assumções que são expressas pelo paciente, assim podendo melhorar a informação para o melhor resultado terapêutico ${ }^{47}$.

No entanto, no atual contexto de correlações de forças, a sociedade civil e a médica continuam a ser orientadas para o consumo, mesmo que irracional e apenas orientado pela necessidade de lucrar, criando a necessidade de tratar farmacologicamente, originando a dúvida sobre a necessidade de tratar e atuando apenas do lado da procura para que haja pedido de prescrição, haja compra de medicamentos e haja uma noção de mistificação de que o medicamento é a fonte da cura, alterando-se, para muitas patologias, a necessidade terapêutica, em particular para as sociais. Ou seja, mais tratar que prevenir. E, se não tratar, estará a mal orientar o caso, pois o conhecimento passará a depender de um interesse de usar tecnologia sem curar, de saber da sua real valia a prazo.

\section{Conclusões}

Pelo exposto e por até hoje não estar estudado este importante campo, julga-se necessário saber o conhecimento dos utilizadores sobre o que é um medicamento, quais os motivos sentidos para o consumo de medicamentos, que conhecimentos são detidos acerca do medicamento no corpo (absorção, distribuição, atuação, metabolização e eliminação), qual o conhecimento acerca do valor econômico do medicamento, bem como saber do acesso a outras formas de terapêutica como a naturopatia, a ervanária e a acupuntura e conhecimento acerca do triângulo médico-doente-medicamento, que será importante contributo para a mais eficiente utilização de medicamentos, sendo certo que esta área é uma das muito sensíveis da Medicina Geral e Familiar.

\section{Referências}

1. Decreto-Lei n.o 176/2006 de 30 de Agosto publicado em Diário da República, 1.a série-N.o 167-30 de
Agosto de 2006.

2. Drhova L. Knowledge, attitudes, and behaviour of the population of the Czech Republic to self-medication. III: behaviour in the area of drugs and self-medication. Ceska Slov Farm. 2005 Sep; 54(5):211-8.

3. Vallerand AH, Fouladbakhsh J, Templin T. Patients' choices for the self-treatment of pain Appl Nurs Res. 2005 May;18(2):90-6.

4. Marques FB, Cobrado N, Caramona M. Caracterização da natureza e dos custos financeiros directos da automedicação. Rev Port Clin Geral 2000;16(1):23-34.

5. Maria VJ Automedicação, custos e saúde. Rev Port Clin Geral 2000;16(1):11-14.

6. Drhova L. Knowledge, attitudes, and behaviour of the population of the Czech Republic toself-medication I: Knowledge of and information on drugs. Ceska Slov Farm. 2005;54(3):123-9.

7. Prevenção Cardiovascular: Recomendações para a Abordagem do Risco Vascular Associado às Dislipidemias. Recomendações da Sociedade Portuguesa de Cardiologia. Rev Port Cardiol 2002; 21(10):1201-1209

8. Diagnóstico, tratamento e controlo da Hipertensão Arterial, Circular Normativa no 2/DGCG de 31 de Março de 2004, Direcção Geral da Saúde, Ministério da Saúde

9. European guidelines on cardiovascular disease prevention in clinical practice: the Third Joint Task Force of European and other Societies on Cardiovascular Disease Prevention in Clinical Practice. European Heart Journal 2003; 24:16011610

10. The seventh report of the Joint National Committee on Prevention, Detection, Evaluation, and Treatment of high Blood Pressure, The JNC 7 Report. Chobanian AV, Bakris GL, Black HR, et al JAMA 2003; 289(19): 2560-2572

11. Schwatrz PJ, Breithardt G, Hioward AJ, Julian DG, Ahlberg NR. The legal implications of medical Guidelinesa Task Force of the European Society of Cardiology. European Heart Journal 1999; 20:1152-1157.

12. Avery AJ, Rodgers S, Heron T, Crombie R, Whynes D, Pringle $\mathrm{M}$ et al. A prescription for improvement? An observational study to identify how general practices vary 
in their growth in prescribing costs. BMJ 2000; 321:276-81 13. Moynihan R, Smith R Demasiada Medicina: quase de certeza. BMJ 2002; 11 345-346

14. Disponível em: http://www.infarmed.pt/portal/page/ portal/infarmed/medicamentos_uso_humano/ prescricao_dispensa_e_utilizacao/medicamentos _e_conducao/folheto_utentes.pdf

15. http://www.infarmed.pt/portal/page/portal/ infarmed/legislacao/legislacao_farmaceutica_compilada/ titulo_ii/titulo_ii_capitulo_VI/portaria_827-2005.pdf

16. http://www.infarmed.pt/portal/page/portal/ infarmed/monitorizacao_do_mercado/observatorio/ analise_mensal_mercado/mnsrm_200608_1.pdf

17.http://www.infarmed.pt/portal/page/portal/ infarmed/publicacoes/tematicos / boletim_ farmacovigilancia/bf10_1.pdf

18. http://medicine.iupui.edu/flockhart/table.htm

19. Santiago LM Interacção entre medicamentos e alimentos. PostGraduate2006; 26(1):69-80

20. World Health Organization: Technical Report no 48. Geneva:1972

21. http://www.opas.org.br/coletiva/uploadArq/AlmaAta.pdf

22. http://www.opas.org.br/coletiva/uploadArq/ Sundsvall.pdf

23. http://www.saudepublica.web.pt/05-PromocaoSaude/ Dec_Jacarta.htm

24. http://www.who.int/healthpromotion/conferencs/ previous/ottawa/enprint.html

25. Dias FN. Fundamentos da manipulação. A manipulação do conhecimento. Comunicação \& linguagens: 39-46

26. http://ec.europa.eu/dgs/health_consumer/events/ future_challenges_paper.pdf

27. http://ec.europa.eu/health/ph_overview/Documents/ ev_20050405_rd01_en.pdf

28. Bryan S. Turner, The Body \& Society. In: Disease and Disorder. [s.l.:s.n]; [s.d]. 197:214.

29.Sousa JC. Do peixe no prato à cana de pesca.Reflexões sobre empoderamento, capacitação e cuidados de saúde. Rev Port Clin Geral 2007;23:353-8
30. Yaphe J. Teaching and learning about empowerment in family medicine. Rev Port Clin Geral 2007;23:365-7

31. Jardim J, Pereira A. Competências pessoais e sociais, guia prático para a mudança positiva. [s.l.]: Edições ASA; [s.d]. 31-36.

32. Gérvas J. Innovación Tecnológica em Medicina: Una Visión Crítica. Rev Port Clin Geral 2006;22:723-7)

33. Galvão C. O envelhecimento e cuidados geriátricos em Medicina Familiar. Rev Port Clin Geral 2006; 22: 729-30.

34. A definição Europeia de Medicina Geral e Familiar. Versão Reduzida - EURACT. Rev Port Clin Geral. 2005; 21:511-516

35. Melo M. A prevenção quaternária contra os excessos da Medicina. Rev Port Clin Geral 2007; 23: 289-293.

36. Saraiva JA. Diálogo. Tabu, Jornal Sol. 46:80-81

37. Santiago LM Interacção entre medicamentos e alimentos. PostGraduate. 2006; 26(1):69-80

38. Santiago LM, Fernandes J, Francisco MP, Neto G, Carvalho IM, Rocha MG, Parra LN. Interacções farmacocinéticas na prescrição - um estudo alargado no ambulatório de Medicina Geral e Familiar na área da Sub-região de Saúde de Coimbra. Rev Port Clin Geral: 323-336

39. Saúde Pública 8, Instituto de Higiene e Medicina Social da Faculdade de Medicina da Universidade de Coimbra, "Determinantes e Condicionantes da Prescrição no Ambulatório de Clínica Geral/Medicina Geral e Familiar na área da Administração Regional de Saúde do Centro de Portugal", Santiago LM: 75-90.

40.http:/ / www.scielosp.org/scielo.php? script=sci_ar ttext\&pid=S0034-89102001000200016\&lng=en\&nrm=iso 41. Macedo ME et al. Prevalence, awareness, treatment and control of hypertension in Portugal: the PAP study. J.Hypertens.2005; 23(9):1661-1669.

42. http://es2005.fe.uc.pt/files/resumos/posters/pf14.pdf 43. http://es2005.fe.uc.pt/files/resumos/posters/pf14.pdf 44. http://www.valormed.pt/images/ficheiros_pdf/ Relatorio_Actividades_INR_2006_2_draft.pdf

45. http://www.nzma.org.nz/journal/115-1162/183/ 46. http://www.ama-assn.org/ama/pub/category/ 13610.html 
47. http:/ /www.diva-portal.org/oru/abstract.xsql?dbid=734

48. Melo M. A promoção da doença. A quem interessa?

Rev Port Clin Geral 2007; 23: 361-4.

\section{Endereço para correspondência:}

Luiz Miguel Santiago

Quinta de Voimarães, lote $12-5^{\circ} \mathrm{D}$

3000-377 - Coimbra

\section{Endereço eletrônico:}

lmsantiago@netcabo.pt 